\title{
Environment monitoring sensor system design based on aircraft
}

\author{
Hong Zhang ${ }^{1, a^{*}}$, Yalin $\mathrm{Li}^{2, \mathrm{~b}}$ \\ ${ }^{1}$ School of physics and information engineering, Jianghan University, Wuhan of China \\ ${ }^{2}$ School of chemistry and environment engineering, Jianghan university, Wuhan of China \\ a1525859704@qq,com, linyali_j07@yahoo.com
}

Keywords: Four axis aircraft; Chemical sensor; Zigbee network; Monitoring network; the host computer

Abstract:In order to do real-time monitoring disaster prediction and life detection of flammable an $\mathrm{d}$ explosive, high risk and highly pressure goods in the factory etc. Combination wireless sensor network node with temperature, humidity ,combustible gas concentration sensor , using four rotor aircraft as a carrier of the monitoring sensor node to the designed region, using decorated wireless sensor network node Zigbee form local area monitoring network by ad-hoc network. The system can not only transmit the sensor node monitoring data to the ground station by the network, but also can use the upper computer to analysis the data.

\section{Introduction}

Aircraft as a kind of intelligence robot in environmental monitoring, security, detection, and other fields has been widely used. With the gradual development of Micro Electrical-Mechanical System (MEMS) ,sensor network and molding technology, wireless sensor network with four axis aircraft apply to the field of environmental monitoring, not only can take advantage of the wireless network dynamic characteristics and ad-hot network, it can also use the performance of the vehicle lightweight flexible to strain various sudden emergencies, realize the performance of supervisory control and data real-time transmission.

\section{Organization of the Text}

Four axis aircraft.Four axis vehicles cross shaped, in four endpoints were fixed with four propeller that the performance is exactly the same, motor rotation provide enough lift to aircraft .At the same time ,the propeller adopt the design of the positive and negative propeller support to offset the torque of propeller rotation.

Working principle.In four axis aircraft, it is equipped with three axis accelerometer and gyroscope sensor, they are consisting of inertial navigation module .It is can calculate the attitude as well as the acceleration, velocity of aircraft relative to the ground at this time. Flight controllers through algorithm calculating the rotation of the force which is to keep the motion state and lift ,through appropriate power to ensure that the motor output. There are two ways for Aircraft fly is shown in Fig.1,six degrees of freedom(change of six direction) is shown in Fig.2,four control input(four motor). So ,the flight attitude is decided by the speed of four propeller, they can realize aircraft pitch ,roll and yaw ,six direction control together.

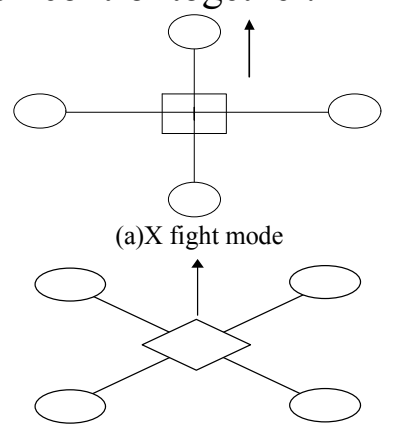

(b)十 fight mode

Fig.1 The way of flight 


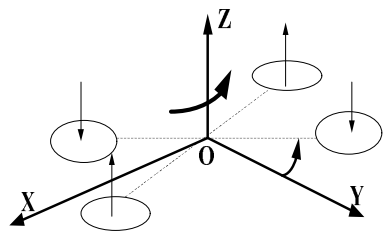

(a) Yaw

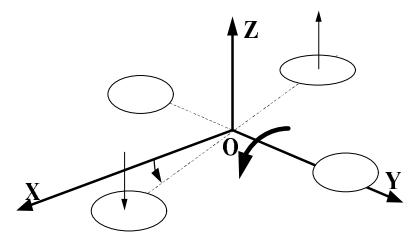

(b) Pitch

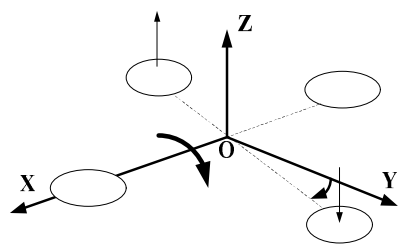

(c) Roll

Fig.2 Flight attitude

System structure.To make four axis aircraft flying stability, the aircraft hardware has three section is shown in Fig. 3, mainly include master chip, gesture detection module, drive module and other major component. In each program :(1)using the high performance, low price and power consumption STM32F103RCT6 32-bit SCM system as the core; (2)using the higher integration of mpu6050 (three-axis gyroscope + three-axial acceleration) measuring aircraft Angle and angle velocity changes on the $\mathrm{X}, \mathrm{Y}, \mathrm{Z}$ axis; (3)using the quaternion, the direction cosine matrix, Euler angles describe the body posture; (4)through the PID(proportion, integral, differential) control algorithm processing spacecraft attitude error and drive motor to maintain vehicle stability; (5)using the power triode SI2302 hollow cup motor to provide enough lift and change the aircraft flight attitude.Effect of flight is shown in Fig.4.

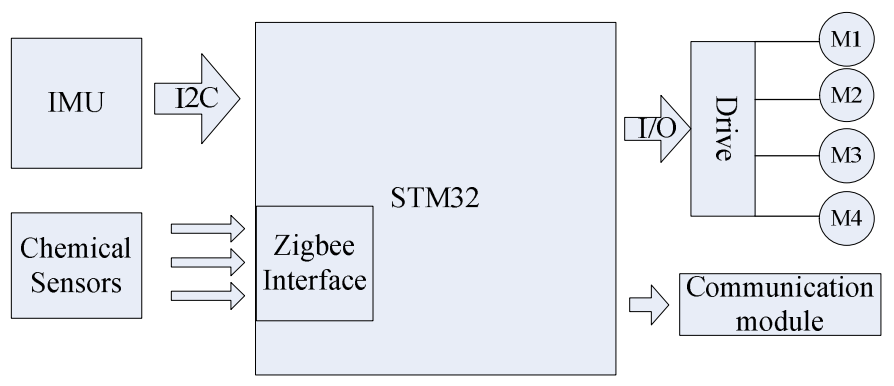

Fig.3 Flight control system hardware connection

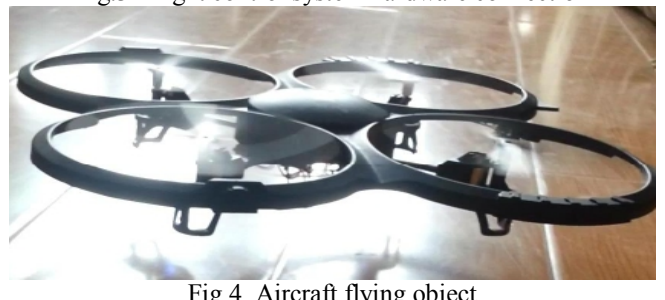

Fig.4 Aircraft flying object

Monitoring network.Using Zigbee technology to monitor all sensor nodes in networking area .This scheme worked in the IAR EW8051 software integrated development environment, it is based on TI/Chipcon company of Zigbee2006 protocol stack development free of charge.TI Z-Stack protocol stack is based on a rotation query type operating system. The main protocol stack process is shown in Fig.5. 


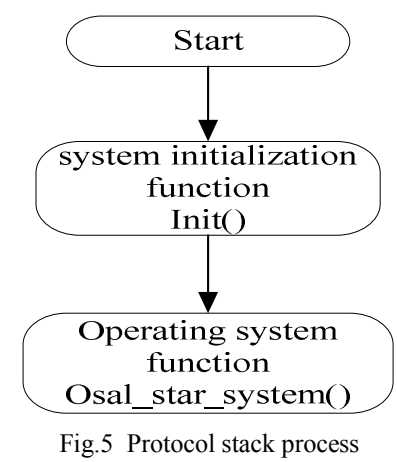

Data delivery.In the process of Zigbee network, throughing the coordinator network sent command. After the success of the networking,periodic send data function be called to send sensor data .

In the system networking,the coordinator send address by broadcase ways, each node in the network begin to monitor and choose to join this network. After the success of the nodes join the network, they will get IDs which is distribed by coordinator.All networking process is shown in Fig.6. Among of them,the coordinator send the address and the frame structure of address is : the device address low 8 and high 8 bit device address.

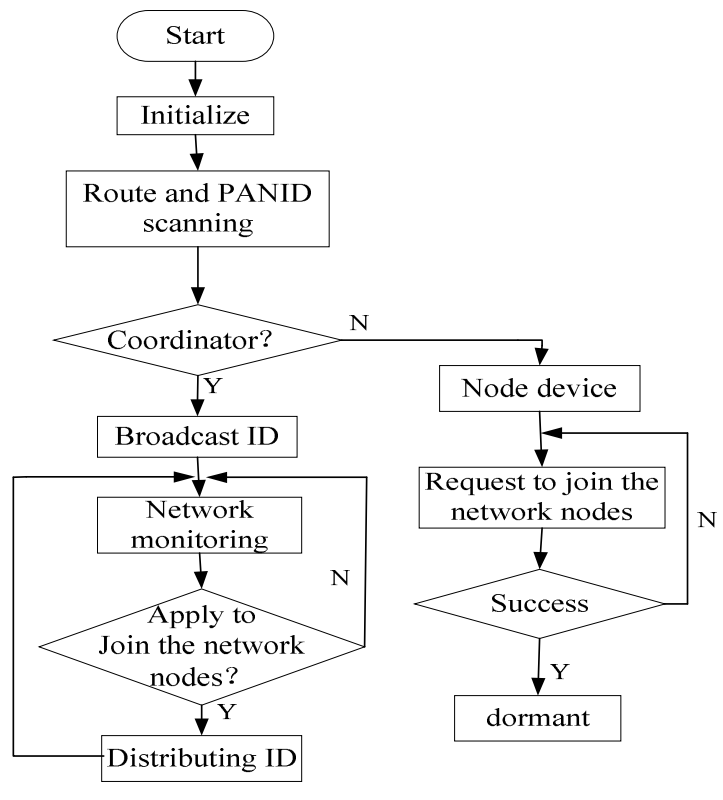

Fig.6 Zigbee Networking process

After each node be assigned address by coordinator includeing aircraft carrying node,each nodes can be targeted for data transmission. As is shown in Fig.7,Zigbee nodes that on the Aircraft can col lect sensor data after the ADC sampling, then operating system Osal invokes the periodic send funct ion SampleApp_SendPeriodicMessage to send.

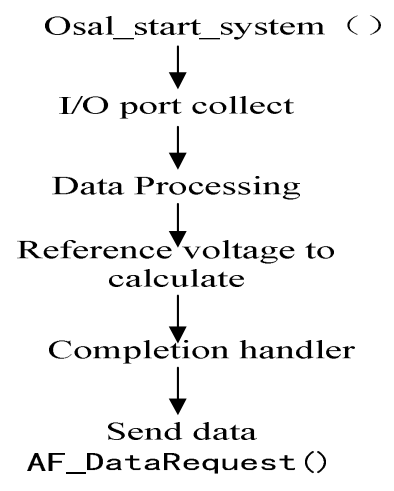

Fig.7 Sensor data sending process 
Data reception.On aircraft Zigbee nodes, it has received the temperature ,humidity,carbon dioxide density data.Each node could through this network transmission data to coordinator and the data will be send to upper computer after coordinator received the data.

In the scheme,the common gas monitoring are using TN901,HR202,MQ-2 sensors. Three kinds of sensor integration module through the figure interface connected with Zigbee nodes I/O is shown in Fig.8.

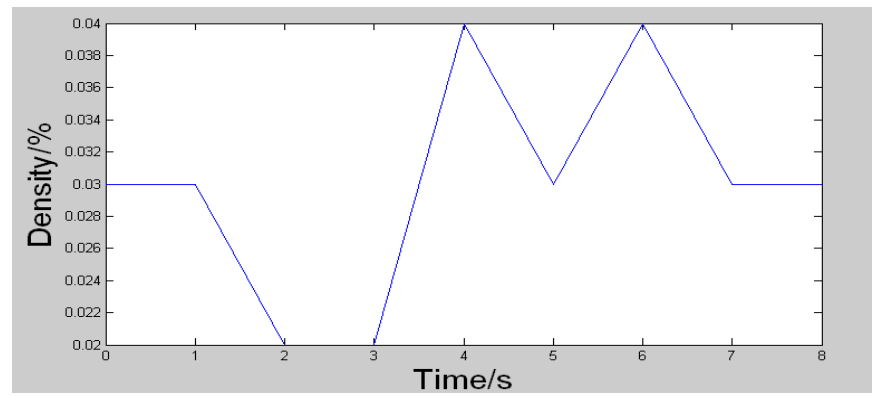

The coordinator default device address $0 \mathrm{x} 0000$ in the process of ad-hoc network, so,aircraft node transfer the sensor data to this address. After data be deliveried to the address of $0 x 0000$, the coordinator operating system Osal function begin receive data by AF_INCOMING_MSG_CMD function, the received data protocol is shown in Table 1, then the data would be send to the serial port by HalUARTWrite function, the data curve is shown in Fig.9.

Table 1 Node data reception frame structure

\begin{tabular}{ccccccc}
\hline Byte0: & 1 & 2 & 3 & 4 & 5 & 6 \\
\hline & High & Low & High & Low & High & Low \\
Identifier & humidity eight & humidity eighth & temperature of eight & temperature of eight & concentration of eight & concentration of eight \\
\hline
\end{tabular}
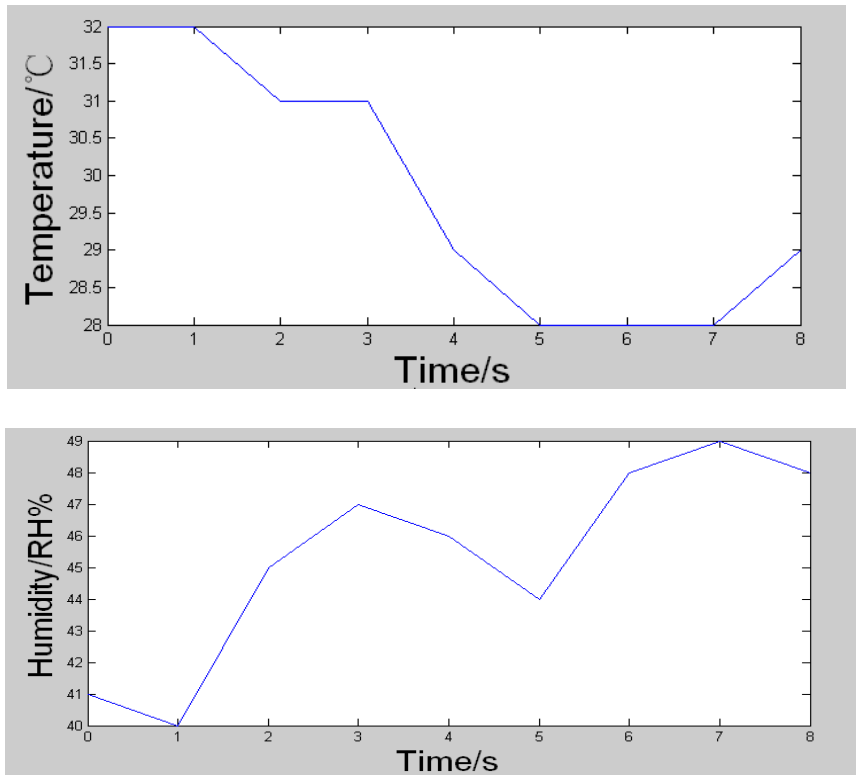

Fig.9 The data of $\mathrm{PC}$ receives

\section{Conclusion}

This system take advantage of four axis aircraft carrying wireless sensor network Zigbee node and chemical sensor, using wireless sensor network delivered data to the monitor in real-time.Finally,the design achieved the expectes effect in the laboratory. Spacecraft successfully tested and make use of the wireless sensor network Zigbee mesh topology of local area network,each terminal node sensor 
acquisition the value successfully and transmitted to the PC interface.The design for extreme environment of environmental monitoring has certain reference significance.

\section{References}

[1] R.O. De Alba, N.S. Ferguson, B.R. Mace. A robust spot weld model for structural vibration analysis[J]. Finite Elements in Analysis \& Design, 2014.

[2]Xiaocong He, Fengshou $\mathrm{Gu}$, Andrew Ball. A review of numerical analysis of friction stir welding[J]. Progress in Materials Science, 2014, 65.

[3] Md Asri Bin Ngadi, Saqib Ali, Abdul Hanan Abdullah et al.. A taxonomy of cross layer routing metrics for wireless mesh networks[J]. EURASIP Journal on Wireless Communications and Networking, 2012, 2012(1).

[4]Tanaka K, Atarashi H, Yamaguchi I et al.. Wireless LAN Security Management with Location Detection Capability in Hospitals.[J]. Methods of Information in Medicine, 2011, 50(3).

[5] Sari Lakkis, Rafic Younes, Mazen Ghandour et al.. New optical gas sensor for gas concentration measurement using digital image processing[J]. Sensors \& Actuators: B. Chemical, 2014. 